\title{
Recent advances and challenges in microneedle-mediated transdermal protein and peptide drug delivery
}

\begin{abstract}
Recent developments in biotechnological techniques such as protein purification and recombinant DNA technology have led to the production of many commercial protein- and peptide-based drugs. Although the intravenous route is the preferred mode of protein drug delivery, it is associated with pain, cost, and sterility concerns. These complications can be avoided with minimally invasive microneedle (MN)-mediated transdermal drug delivery to provide better patient compliance. Currently, various formulation and microfabrication techniques are being developed to facilitate $\mathrm{MN}$-mediated protein drug delivery. The design of MNs primarily relies on the heterogeneous and viscoelastic property of the skin. Presently, several MN brands have been successfully marketed and others are actively undergoing clinical trials to confirm their efficacy in the delivery of drug candidates. This chapter mainly highlights the latest developments and limitations in MN-mediated protein and peptide drug delivery. The proposed mechanism of entry of drugs administered via $\mathrm{MN}$ into blood circulation also has been discussed.
\end{abstract}

Keyword: Microneedles; Transdermal drug delivery; Minimally invasive; Protein; Peptide 